\title{
ESTUDO COMPARATIVO DE DELINEAMENTOS EXPERIMENTAIS PARA ESTIMATIVAS DE PARÂMETROS GENÉTICOS EM ERVA-MATE (Ilex paraguariensis A. St. - Hil.) ${ }^{1}$
}

\author{
José Sebastião Cunha Fernandes², Marcos Deon Vilela de Resende ${ }^{3}$, José Alfredo Sturion ${ }^{3}$, Agenor \\ Maccari Jr. ${ }^{4}$
}

\begin{abstract}
RESUMO - O presente trabalho teve por objetivo comparar a eficiência do látice em relação aos delineamentos em blocos casualizados (DBC) e inteiramente casualizados (DIC) quanto aos caracteres altura e produção de massa verde em erva-mate (Ilex paraguariensis A. St. - Hil.). O experimento, conduzido na Fazenda Experimental do Canguiri, da Universidade Federal do Paraná, em Pinhais, PR, foi composto de cinco procedências, quatro com 13 e uma com 12 progênies de meias-irmãs. Cada progênie continha 54 plantas (nove parcelas de seis plantas). O experimento seguiu o modelo látice $8 \times 8$ com nove repetições balanceadas. As análises foram conduzidas usando-se tanto este modelo quanto os modelos DBC e DIC. Com relação ao caráter altura, a estimativa da eficiência do DBC em relação ao DIC ( $\hat{\mathrm{E}}_{\mathrm{b} / \mathrm{i}}$ ) foi de $48,6 \%$; do látice em relação ao DBC $\left(\hat{\mathrm{E}}_{1 / \mathrm{b}}\right)$, de $61 \%$; e do látice em relação ao DIC $\left(\hat{\mathrm{E}}_{1 \mathrm{i}}\right)$, de $139 \%$. A estimativa da correlação intraclasse entre plantas dentro das parcelas $\left(\hat{\mathrm{c}}^{2}\right)$ foi de $9,4 \%$ para o DBC e zero para o látice. Para o caráter produção de massa verde, $\hat{\mathrm{E}}_{\mathrm{b} / i}$, $\hat{\mathrm{E}}_{1 / \mathrm{b}}$ e $\hat{\mathrm{E}}_{1 / \mathrm{i}}$ foram de 14,$3 ; 81,7$; e $108 \%$, respectivamente; e $\hat{\mathrm{c}}^{2}, 16,9 \%$ para o DBC e $3,1 \%$ para o látice. Essas estimativas, associadas a uma estimativa de $\mathrm{F}$ de Snedecor altamente significativa dos dois caracteres, permitiram concluir que a capacidade de teste do DBC foi satisfatória para o caráter altura de plantas, mas insatisfatória para o caráter produção de massa verde.
\end{abstract}

Palavras-chave: Heterogeneidade ambiental, blocos incompletos, herdabilidade e blocos ao acaso.

\section{EXPERIMENTAL DESIGNS TO ESTIMATE GENETIC PARAMETERS IN MATÉ (Ilex paraguariensis A. St. - Hil.)}

\begin{abstract}
The aim of the present work was to compare the efficiency of the lattice design with Random Block Design (RBD) and the latter with Completely Randomized Design (CRD), for plant height and fresh matter weight (biomass) traits in mate (Ilex paraguariensis A. St. - Hil.). The trial was performed at the Estação Experimental do Canguiri, Universidade Federal do Paraná, in Pinhais, Paraná State. Out of 64 half-sib progenies, 52 came from four different sites, 13 from each site, in the State of Paraná and 12 from a site in the State of Rio Grande do Sul. Each progeny consisted of 54 plants (nine plots with six plants per plot). An $8 \times 8$ lattice design model with nine balanced repetitions was used to carry out the trial. The analyses were performed also using RBD and CRD models. For plant height, the estimation of RBD efficiency compared to $C R D\left(\hat{E}_{b / i}\right)$; lattice compared to $R B D\left(\hat{E}_{l / b}\right)$; and lattice compared to $C R D\left(\hat{E}_{l / i}\right)$ were $48,6 \%$; $61,0 \%$; and $139 \%$, respectively. The estimation of the intraclass correlation among plants within plots $\left(\hat{\mathrm{c}}^{2}\right)$ was $9,4 \%$ and zero for $R B D$ and lattice, respectively. For fresh matter, $\hat{E}_{b / /}, \hat{E}_{l / b}$ and $\hat{E}_{l / i}$ were $14,3 \%, 81,7 \%$ and $108 \%$, respectively and $\hat{\mathrm{c}}^{2}$ were $16,9 \%$ for $\mathrm{RDB}$ and $3,1 \%$ for lattice. These estimations coupled with Snedecor's F tests showed high significance for the two traits, indicating efficiency of RBD in relation to $C R D$ greater for plant height than for fresh matter.
\end{abstract}

Key words: environment heterogeneity; incomplete blocks; heritability; mate.

\footnotetext{
${ }^{1}$ Recebido para publicação em 08.9.2003 e aceito para publicação em 10.8.2004.

${ }^{2}$ FAFEID, Diamantina, MG. E-mail: <cunha@citel1.com.br>.

${ }^{3}$ Embrapa Florestas, Colombo, PR.

${ }^{4}$ Departamento de Solos e Engenharia Agrícola/UFPR, Curitiba, PR.
} 


\section{INTRODUÇÃO}

Estimativas de parâmetros genéticos têm sido largamente empregadas pelos melhoristas para melhor conhecerem o potencial genético de indivíduos, famílias e clones, entre outros, a serem selecionados e, ou, recombinados para um novo ciclo de seleção.

A estimação desses parâmetros requer delineamentos genético estatísticos fundamentados em modelos matemáticos. Estes últimos determinam a maneira como serão montados os experimentos, bem como os possíveis procedimentos estatísticos relacionados à estimação de parâmetros.

O Delineamento em Blocos Casualisados (DBC) tem sido freqüentemente utilizado no melhoramento genético vegetal. Seu modelo leva em consideração o efeito $b_{j}$, que pode ser isolado dos demais na análise.

No Delineamento Inteiramente Casualizado (DIC), de uso bem menos freqüente entre os melhoristas, partese do pressuposto de que toda a área ou ambiente experimental seja suficientemente uniforme,de maneira que a estratificação é suprimida.

Quando ocorre, entretanto, o fato de que a uniformidade dentro dos blocos passa a ser comprometida, seja em razão do excessivo número de tratamentos, seja por causa da heterogeneidade do ambiente experimental, necessário se faz dividir os blocos em subunidades menores. Como estas subunidades não recebem todos os tratamentos, são denominadas blocos incompletos, mais usados em experimentos com grande número de tratamentos.

Dos delineamentos em blocos incompletos, os mais empregados são os blocos incompletos equilibrados "balanced incomplete blocks" e os reticulados quadrados "square lattices" ou "two dimensional lattices" (PIMENTEL GOMES, 2000).

Informações sobre DIC e DBC são comuns em vários compêndios (FEDERER, 1955; COCHRAN e COX, 1957; VIEIRA e HOFFMANN, 1989; VENCOVSKY e BARRIGA, 1992; CRUZeREGAZZI, 1994; PIMENTEL GOMES, 2000; RAMALHO et al., 2000; RESENDE, 2002), bem como em diversos artigos. Esses delineamentos, principalmente o DBC, são amplamente disseminados nas ciências agrárias, biológicas e da saúde.

R. Árvore, Viçosa-MG, v.28, n.5, p.663-671, 2004
Os blocos incompletos e em particular os reticulados quadrados são, em relação ao DBC e DIC, menos explorados nos compêndios. Seu uso é também bem menos disseminado, talvez devido a dificuldades na montagem, análise e interpretação dos resultados.

No melhoramento florestal, o DBC tem sido o mais utilizado na América do Norte (FU et al., 1998), sendo os blocos incompletos os de uso mais comum na Austrália, África do Sul e Ásia (WILLIAMS e MATHESON, 1994). No Brasil há indícios do uso em iguais proporções desses dois delineamentos (RESENDE e FERNANDES, 2000).

Um aspecto importante, diante do que se apresenta, é a comparação da eficiência desses três delineamentos. Segundo Resende e Fernandes (2000), a eficácia do melhoramento genético para caracteres quantitativos depende da utilização de delineamentos experimentais também eficazes. No entanto, esses autores mencionaram também o nível de variação ambiental como determinante importante na eficiência relativa entre os delineamentos.

Resende (2002) propôs a combinação do coeficiente de correlação intraclasse entre plantas dentro das parcelas $\left(\mathrm{c}^{2}\right)$ com o teste $\mathrm{F}$ de Snedecor para blocos, para se inferir sobre a eficiência e capacidade de teste de um delineamento, com quatro possibilidades: $\mathrm{c}^{2}$ alto $(>10 \%)$ e baixo $(<10 \%)$, combinado com F significativo e não-significativo para blocos. A situação ideal seria a combinação de um $\mathrm{c}^{2}<10 \%$ (estratos homogêneos) com um F significativo para blocos (grande efeito entre os estratos sobre o caráter avaliado).

O presente trabalho teve por objetivo comparar a eficiência desses três delineamentos estatísticos no melhoramento genético da erva-mate.

\section{MATERIAL E MÉTODOS}

O material, mudas de erva-mate, originado de quatro procedências do Paraná (Ivaí, Quedas do Iguaçu, Colombo e Cascavel), com 13 progênies cada, e de uma do Rio Grande do Sul (Barão de Cotegipe), com 12 progênies, foi cedido pelo Centro Nacional de Pesquisas de Florestas (CNPF) da Empresa Brasileira de Pesquisa Agropecuária (EMBRAPA) à Universidade Federal do Paraná (UFPR).

O experimento foi montado em uma área declivosa, sendo as repetições estabelecidas no sentido de 
uniformizar seus níveis. Contudo, notaram-se ainda diferenças de nível dentro das repetições. Amostras de solo foram retiradas para análises químicas em três estratos ao longo de cada repetição e duas profundidades para cada extrato, totalizando 54 amostras, cada uma composta de 18 subamostras, totalizando 972 subamostras (FERNANDES et al., 2000).

Cada progênie ou família compreendia cerca de 60 plantas ( 54 foram usadas no plantio e as demais, na reposição de falhas), supostamente meias-irmãs, já que a espécie é dióica, de polinização principalmente entomófila, e com grande dispersão de pólen (WINGE et al., 1995).

O experimento foi implementado em látice 8 × 8 (64 famílias, 52 oriundas em iguais quantidades dos municípios paranaenses de Colombo, São Mateus do Sul, Ivaí e Cascavel, e 12 oriundas do município de Barão de Cotegipe, RS) com nove repetições balanceadas e seis plantas por parcela (54 plantas por progênie), perfazendo um total de 3.456 plantas. Foi estabelecido em agosto de 1997, no município de Pinhais, PR, na Estação Experimental do Canguiri, pertencente à Universidade Federal do Paraná (UFPR). O caráter altura de planta foi analisado um ano após o plantio e o peso de massa verde (biomassa), três anos após. As mudas foram transplantadas com 1,5 ano de idade.

Os modelos matemáticos considerados nos três delineamentos foram: a) para o DIC: $\mathrm{y}_{\mathrm{ijk}}=\mu+\mathrm{f}_{\mathrm{i}}+\mathrm{e}_{\mathrm{i} / \mathrm{j}}+\mathrm{d}_{\mathrm{ijk}}$, em que $y_{\mathrm{ijk}}=$ observação no indivíduo ijk; $\mu=$ média geral, fixa, $\mathrm{E}(\mu)=\mu$ e $\mathrm{E}(\mu)^{2}=\mu^{2} ; \mathrm{f}_{\mathrm{i}}=$ efeito da família i dentro de procedências, aleatório, $\mathrm{E}\left(\mathrm{f}_{\mathrm{i}}\right)=0$ e $\mathrm{E}\left(\mathrm{f}_{\mathrm{i}}^{2}\right)=\sigma_{\mathrm{f}}^{2}$; $\mathrm{e}_{\mathrm{i} / \mathrm{j}}=$ efeito da parcela $\mathrm{ij}$ dentro da família $\mathrm{i}$ (erro do DIC), aleatório, $\mathrm{E}\left(\mathrm{e}_{\mathrm{i} / \mathrm{j}}\right)=0$ e $\mathrm{E}\left(\mathrm{e}_{\mathrm{i} / \mathrm{j}}^{2}\right)=\sigma^{2}$; e $\mathrm{d}_{\mathrm{ijk}}=$ efeito do indivíduo ijk, aleatório, $E\left(d_{i j k}\right)=0$ e $E\left(d_{i j k}^{2}\right)=\sigma_{d}^{2}$. b) para o DBC: $y_{i j k}=\mu+f_{i}+r_{j}+e_{i j}+d_{i j k}$, sendo $r_{j}=$ efeito da repetição $\mathrm{j}$, aleatório, $\mathrm{E}\left(\mathrm{r}_{\mathrm{j}}\right)=0$ e $\mathrm{E}\left(\mathrm{r}_{\mathrm{j}}^{2}\right)=\sigma_{\mathrm{r}}^{2}$; e e $\mathrm{e}_{\mathrm{ij}}=$ efeito da parcela ij (erro do DBC), aleatório, $\mathrm{E}\left(\mathrm{e}_{\mathrm{ij}}\right)=0$ e $\mathrm{E}\left(\mathrm{e}_{\mathrm{ij}}^{2}\right)=\sigma_{\mathrm{e}}^{2}$, sendo os demais efeitos iguais aos do DIC. c) para o látice: $\mathrm{y}_{\mathrm{ijlk}}=\mu+\mathrm{f}_{\mathrm{i}}+\mathrm{r}_{\mathrm{j}}+\mathrm{b}_{\mathrm{j} / \mathrm{l}}+\mathrm{e}_{\mathrm{ijl}}+\mathrm{d}_{\mathrm{ijlk}}$, em que $\mathrm{b}_{\mathrm{j} / \mathrm{l}}$ é o efeito do bloco 1 dentro da repetição $\mathrm{j}$, aleatório, $\mathrm{E}\left(\mathrm{b}_{\mathrm{j} / 1}\right)=0$ e $\mathrm{E}\left(\mathrm{b}_{\mathrm{j} / 1}^{2}\right)=\sigma_{\mathrm{b}}^{2} ;$ e e $_{\mathrm{ijl}}$ é o erro intrabloco, aleatório, $\mathrm{E}\left(\mathrm{e}_{\mathrm{ij}}\right)=0$ e $E\left(e_{i j l}^{2}\right)=\sigma_{\text {eib }}^{2}$, sendo os demais efeitos iguais aos anteriores. Em todos os modelos, o efeito $f_{i}$ refere-se ao efeito de famílias dentro de procedências. Os índices $\mathrm{i}, \mathrm{j}$, k e 1 variam de 1 a $\mathrm{I}=64$, de 1 a $\mathrm{J}=9$, de 1 a $\mathrm{K}=6$ e de 1 a $\mathrm{L}=8$, respectivamente. Como houve perda de plantas em várias parcelas, $\mathrm{K}$ foi tomado como a média harmônica $(\overline{\mathrm{k}})$ do número de plantas por parcela.

Os quadros das análises de variância (anovas) de cada modelo, em nível de média de parcela, com as respectivas esperanças dos quadrados médios, são mostrados na Tabela 1 .

A eficiência do DBC em relação ao DIC $\left(\mathrm{E}_{\mathrm{b} / \mathrm{i}}\right)$ foi obtida pelo quociente $\bar{\sigma}^{2} / \bar{\sigma}_{\mathrm{e}}^{2}=\left(\bar{\sigma}_{\mathrm{e}}^{2}+\sigma_{\mathrm{r}}^{2}\right) / \bar{\sigma}_{\mathrm{e}}^{2}$. A eficiência do látice em relação ao $\operatorname{DBC}\left(\mathrm{E}_{1 / \mathrm{b}}\right)$ foi obtida pelo quociente $\bar{\sigma}_{\mathrm{e}}^{2} / \bar{\sigma}_{\text {eib }}^{2}=\left(\bar{\sigma}_{\text {eib }}^{2}+\sigma_{\mathrm{b}}^{2}\right) / \bar{\sigma}_{\text {eib }}^{2}$. Outros parâmetros estimados foram: o F de Snedecor para o efeito de blocos, já indicados nos quadros das anovas; a correlação intraclasse entre plantas devido ao efeito comum da parcela representado por $\mathrm{c}^{2}=\sigma_{\mathrm{e}}^{2} /\left(\sigma_{\mathrm{d}}^{2}+\sigma_{\mathrm{e}}^{2}+\sigma_{\mathrm{f}}^{2}\right)$ para o DBC e $=\sigma_{\text {eib }}^{2} /\left(\sigma_{\mathrm{d}}^{2}+\sigma_{\text {eib }}^{2}+\sigma_{\mathrm{f}}^{2}\right)$ para o látice; a correlação intraclasse entre parcelas devido ao efeito comum das repetições representado por $\rho_{\mathrm{r}}=\sigma_{\mathrm{r}}^{2} /\left(\sigma_{\mathrm{r}}^{2}+\bar{\sigma}_{\mathrm{e}}^{2}\right)$ para o DBC e $=\sigma_{b}^{2} /\left(\sigma_{b}^{2}+\bar{\sigma}_{\text {eib }}^{2}\right)$ para o látice; a correlação genética intraclasse entre parcelas de um mesmo tratamento (progênie) devido ao efeito desta última, representada por $\rho_{\mathrm{g}}=\sigma_{\mathrm{f}}^{2} /\left(\sigma_{\mathrm{f}}^{2}+\bar{\sigma}^{2}\right) ; \sigma_{\mathrm{f}}^{2} /\left(\sigma_{\mathrm{f}}^{2}+\bar{\sigma}_{\mathrm{e}}^{2}\right) ; \mathrm{e}$ $\sigma_{\mathrm{f}}^{2} /\left(\sigma_{\mathrm{f}}^{2}+\bar{\sigma}_{\text {eib }}^{2}\right)$ para o DIC, DBC e látice, respectivamente; e a correlação genética intraclasse entre as médias das $\mathbf{J}$ parcelas de uma progênie, representada por $\rho_{\bar{g}}=\sigma_{f}^{2} /\left(\sigma_{f}^{2}+\frac{\bar{\sigma}^{2}}{J}\right), \sigma_{f}^{2} /\left(\sigma_{f}^{2}+\frac{\bar{\sigma}_{e}^{2}}{J}\right)$ e; $\sigma_{f}^{2} /\left(\sigma_{f}^{2}+\frac{\bar{\sigma}_{\text {eib }}^{2}}{J}\right)$ para o DIC, DBC e látice, respectivamente. $\rho_{\mathrm{g}}$ pode ser dado também pela expressão $\frac{\mathrm{J} \rho_{\mathrm{g}}}{1+(\mathrm{J}-1) \rho_{\mathrm{g}}}$, usando-se o $\rho_{\mathrm{g}}$ respectivo em cada delineamento.

Foram obtidas também estimativas (médias e coeficientes de regressão) pertinentes à natureza química do solo onde foi conduzido o experimento.

O procedimento para estimar as médias por repetição das variáveis indicadoras de fertilidade foi: cada repetição foi dividida em três estratos (posições inicial, mediana e final). A média de cada variável em cada repetição foi oriunda de seis amostras (três estratos e duas profundidades, 0 a $20 \mathrm{~cm}$ e 20 a $40 \mathrm{~cm}$, por posição). Cada amostra foi composta de 18 subamostras.

Para estimar as médias dessas mesmas variáveis por estrato, procedeu-se da seguinte forma: cada estrato envolveu nove repetições. A média de cada variável em cada estrato foi oriunda de 18 amostras (nove repetições e duas profundidades, 0 a $20 \mathrm{~cm}$ e 20 a 40 $\mathrm{cm}$, por repetição).

R. Árvore, Viçosa-MG, v.28, n.5, p.663-671, 2004 
Cada coeficiente de regressão dos caracteres altura e massa verde foi estimado a partir de 64 pares de dados onde a variável dependente foi a média do caráter na parcela e a independente, a posição (1 a 64) da referida parcela na respectiva repetição.

\section{3) RESULTADOS E DISCUSSÃO}

\subsection{Caráter altura de planta}

As anovas, segundo os delineamentos DIC, DBC e látice, encontram-se nas Tabelas 2,3 e 4 , respectivamente. As estimativas de $E_{b / i}, E_{1 / b}, c^{2}, \rho_{r}, \rho_{g}$, e $\rho_{\bar{g}}$ econtram-se na Tabela 5 .

Como se pode observar na Tabela 5, o DBC teve uma eficiência em relação ao DIC de $49 \%$ e o látice, em relação ao DBC, de $61 \%$ para o referido caráter. A eficiência do látice em relação ao DIC é $\hat{\bar{\sigma}}^{2} / \bar{\sigma}_{\text {eib }}^{2}=2,39$, o que pode também ser obtido pelo produto $1,486 \mathrm{x}$ 1,610. Essas eficiências podem também ser obtidas diretamente a partir de $\hat{\rho}_{\mathrm{r}}$, através da expressão $1 /\left(1-\hat{\rho}_{\mathrm{r}}\right)$, por meio da qual se observa que, quanto maior $\hat{\rho}_{\mathrm{r}}$, maior a eficiência relativa do delineamento. Verificou-se que houve resposta à estratificação da área nos dois níveis (repetições dentro do experimento e blocos dentro de repetições).

Além das eficiências relativas altas para cada um dos dois delineamentos em relação aos imediatamente inferiores no nível de controle local, suas eficiências absolutas foram satisfatórias. Isso pode ser inferido a partir da estimativa $\hat{c}^{2}$ (Tabela 5). Observou-se que ambas estavam abaixo de $10 \%$, sendo nula para o látice. Isso implica já ter havido uma homogeneidade satisfatória dentro das repetições, mas ainda passível de aumento através da estratificação desses delineamentos em estratos menores.

Inferências mais sugestivas podem ser extraídas de uma análise envolvendo informações da eficiência absoluta de um delineamento e da sua capacidade de teste, aspecto esse já abordado na introdução.

Tabela 1 - Análise de variância dos delineamentos inteiramente casualizados (DIC), blocos ao acaso (DBC) e látice em nível de média de parcelas

Table 1 - ANOVA for completely randomized (CRD), randomized block (RBD) and lattice designs at a plot mean base

\begin{tabular}{|c|c|c|c|c|}
\hline \multicolumn{5}{|l|}{ DIC (CRD) } \\
\hline $\mathrm{FV}$ & QM & $\mathrm{E}(\mathrm{QM})$ indivíduos & $\mathrm{E}(\mathrm{QM})$ média & $\mathrm{F}$ \\
\hline Famílias/Procedências & Q1 & $\sigma_{\mathrm{d}}^{2} / \mathrm{k}+\sigma^{2}+\mathrm{J} \sigma_{\mathrm{f}}^{2}$ & $\sigma^{2}+J \sigma_{f}^{2}$ & Q1/Q2 \\
\hline Erro & Q2 & $\sigma_{\mathrm{d}}^{2} / \mathrm{k}+\sigma^{2}$ & $\sigma^{2}$ & Q2/Q3 \\
\hline Dentro & Q3 & $\sigma_{\mathrm{d}}^{2} / \mathrm{k}$ & & \\
\hline \multicolumn{5}{|l|}{ DBC (RDB) } \\
\hline FV & QM & $\mathrm{E}(\mathrm{QM})$ indivíduos & $\mathrm{E}(\mathrm{QM})$ média & $\mathrm{F}$ \\
\hline Repetições & Q1 & $\sigma_{\mathrm{d}}^{2} / \mathrm{k}+\sigma_{\mathrm{e}}^{2}+\mathrm{I} \sigma_{\mathrm{r}}^{2}$ & $\bar{\sigma}_{\mathrm{e}}^{2}+\mathrm{I} \sigma_{\mathrm{r}}^{2}$ & Q1/Q3 \\
\hline Famílias/Procedências & Q2 & $\sigma_{\mathrm{d}}^{2} / \mathrm{k}+\sigma_{\mathrm{e}}^{2}+\mathrm{J} \sigma_{\mathrm{f}}^{2}$ & $\sigma_{\mathrm{e}}^{2}+\mathrm{J} \sigma_{\mathrm{f}}^{2}$ & Q2/Q3 \\
\hline Erro & Q3 & $\sigma_{\mathrm{d}}^{2} / \mathrm{k}+\sigma_{\mathrm{e}}^{2}$ & $\bar{\sigma}_{\mathrm{e}}^{2}$ & Q3/Q4 \\
\hline Dentro & Q4 & $\sigma_{\mathrm{d}}^{2} / \mathrm{k}$ & & \\
\hline \multicolumn{5}{|l|}{ Látice (Lattice) } \\
\hline FV & QM & $\mathrm{E}(\mathrm{QM})$ indivíduos & $\mathrm{E}(\mathrm{QM})$ média & $\mathrm{F}$ \\
\hline Repetições & Q1 & $\sigma_{\mathrm{d}}^{2} / \mathrm{k}+\sigma_{\mathrm{e}}^{2}+\mathrm{I} \sigma_{\mathrm{r}}^{2}$ & $\bar{\sigma}_{\mathrm{eib}}^{2}+\mathrm{I} \sigma_{\mathrm{r}}^{2}$ & Q1/Q4 \\
\hline Famílias/Procedências (ajustado) & Q2 & $\sigma_{\mathrm{d}}^{2} / \mathrm{k}+\sigma_{\mathrm{e}}^{2}+\mathrm{J} \sigma_{\mathrm{f}}^{2}$ & $\bar{\sigma}_{\text {eib }}^{2}+J \sigma_{f}^{2}$ & $\mathrm{Q} 2 / \mathrm{Q} 4$ \\
\hline Blocos/Repetições (ajustado) & Q3 & $\sigma_{\mathrm{d}}^{2} / \mathrm{k}+\sigma_{\mathrm{eib}}^{2}+\mathrm{L} \sigma_{\mathrm{b}}^{2}$ & $\bar{\sigma}_{\mathrm{eib}}^{2}+\mathrm{L} \sigma_{\mathrm{b}}^{2}$ & Q3/Q4 \\
\hline Erro intrabloco & Q4 & $\sigma_{\mathrm{d}}^{2} / \mathrm{k}+\sigma_{\mathrm{eib}}^{2}$ & $\bar{\sigma}_{\text {eib }}^{2}$ & Q4/Q5 \\
\hline Dentro & Q5 & $\sigma_{\mathrm{d}}^{2} / \mathrm{k}$ & & \\
\hline
\end{tabular}

E(QM) média representa a esperança de quadrado médio de uma análise conduzida em nível de média de parcelas; E(QM) indivíduos idem, incluindo-se a variância entre indivíduos dentro das parcelas (variância dentro). 
Tabela 2 - Analise de variância da altura de planta em ervamate com um ano de idade, considerando-se o delineamento inteiramente casualizado (DIC)

Table 2-ANOVA for plant height trait in maté at one year old in a completely randomized design (CRD)

\begin{tabular}{lcccr}
\hline FV & GL & QM & F & P(F) \\
\hline Famílias/Procedências & 59 & 139,9 & 2,3 & $0,00 \%$ \\
Erro & 512 & 59,6 & 2,4 & $0,00 \%$ \\
Dentro & 2550 & 24,9 & & \\
\hline \hline
\end{tabular}

$\overline{\mathrm{k}}=5,43 ; \hat{\sigma}_{\mathrm{d}}^{2}=135,2 ; \hat{\sigma}^{2}=34,7 ; \hat{\sigma}_{\mathrm{f}}^{2}=8,9 ; \hat{\mathrm{h}}_{\mathrm{i}}^{2}=19,9 \%$

Tabela 3 - Análise de variância do caráter altura de planta em erva-mate com um ano de idade, considerandose o delineamento em blocos ao acaso (DBC)

Table 3-ANOVA for plant height trait in maté at one year old in a randomized block design ( $R B D)$

\begin{tabular}{lcccc}
\hline FV & GL & QM & F & P(F) \\
\hline Repetições & 8 & 1289,1 & 32,1 & $0,00 \%$ \\
Famílias/Procedências & 59 & 139,9 & 3,5 & $0,00 \%$ \\
Erro & 504 & 40,1 & 1,6 & $0,00 \%$ \\
Dentro & 2550 & 24,9 & & \\
\hline
\end{tabular}

$\overline{\mathrm{k}}=5,43 ; \hat{\sigma}_{\mathrm{d}}^{2}=135,2 ; \hat{\sigma}_{\mathrm{e}}^{2}=15,2 ; \hat{\sigma}_{\mathrm{f}}^{2}=11,1 ; \hat{\mathrm{h}}_{\mathrm{i}}^{2}=27,5 \% ; \hat{\sigma}_{\mathrm{r}}^{2}=19,5$

Tabela 4 - Análise de variância do caráter altura de planta em erva-mate com um ano de idade, considerandose o delineamento em látice

Table 4-ANOVA for plant height trait in maté at one year old in a lattice design

\begin{tabular}{lcccr}
\hline $\mathrm{FV}$ & $\mathrm{GL}$ & $\mathrm{QM}$ & $\mathrm{F}$ & $\mathrm{P}(\mathrm{F})$ \\
\hline Repetições & 8 & 1289,1 & 51,8 & $0,00 \%$ \\
Famílias/ & 59 & 120,2 & 4,8 & $0,00 \%$ \\
Procedências (aj.) & & & & \\
Bloco/Repetições (aj.) & 63 & 146,8 & 5,9 & $0,00 \%$ \\
Erro intrabloco & 441 & 24,9 & 1,0 & $100,00 \%$ \\
Dentro & 2550 & 24,9 & & \\
\hline$\overline{\mathrm{k}}=5,43 ; \hat{\sigma}_{\mathrm{d}}^{2}=135,2 ; \hat{\sigma}^{2}=0,0 ; \hat{\sigma}_{\mathrm{f}}^{2}=10,6 ; \hat{\mathrm{h}}_{\mathrm{i}}^{2}=29,1 \% ; \hat{\sigma}_{\mathrm{b}}^{2}=15,2$ & &
\end{tabular}

Tabela 5 - Estimativas de $\mathrm{E}_{\mathrm{b} / \mathrm{i}} ; \mathrm{E}_{\mathrm{l} / \mathrm{b}} ; \mathrm{c}^{2} ; \rho \mathrm{r} ; \rho \mathrm{g}$; e $\rho_{\overline{\mathrm{g}}}$ do caráter altura de planta em erva-mate com um ano de idade Table 5 - Estimations for $E_{b / i} ; E_{l / b} ; c^{2} ; \rho r ; \rho g$; and $\rho_{\overline{\mathrm{g}}}$ parameters for plant height in mate trait at one year old

\begin{tabular}{lcccccc}
\hline \multicolumn{5}{c}{ Parâmetros estimados } \\
\hline Delineamentos & $\hat{E} \mathrm{~b} / \mathrm{I}$ & $\hat{\mathrm{E}} \mathrm{l} / \mathrm{b}$ & $\hat{\mathrm{c}}^{2}$ & $\hat{\rho}_{\mathrm{r}}$ & $\hat{\rho}_{\mathrm{g}}$ & $\hat{\rho}_{\overline{\mathrm{s}}}$ \\
DIC & - & - & - & - & 0,130 & 0,573 \\
DBC & 1,486 & - & 0,094 & 0,327 & 0,217 & 0,714 \\
Látice & - & 1,610 & 0 & 0,379 & 0,300 & 0,793 \\
\hline
\end{tabular}

A combinação de uma estimativa de $\mathrm{F}$ para repetições altamente significativas, com uma estimativa de $\mathrm{c}^{2}$ baixa (homogeneidade dentro das repetições), permitiu concluir que o DBC foi não só eficiente, como também propiciou às progênies desenvolverem-se em diferentes condições ambientais, permitindo, neste último caso, a seleção de material com maior plasticidade fenotípica (RESENDE, 2002).

Os valores das estimativas $\hat{\rho}_{\mathrm{g}}$ e $\hat{\rho}_{\overline{\mathrm{g}}}$ aumentam à medida que também aumenta o aprimoramento do delineamento (Tabela 5). Ressaltando-se que $\rho_{\bar{g}}=\frac{J \rho_{\mathrm{g}}}{1+(\mathrm{J}-1) \rho_{\mathrm{g}}}$, em que se verifica que $\rho_{\bar{g}}$ é função de $\rho_{\mathrm{g}} \mathrm{e}$, portanto, correlacionados, o que pode ser constatado também na Tabela 5. Na realidade, $\rho_{\bar{g}}$ aproxima-se da unidade (100\%) à medida que aumenta o número de repetições do experimento. Assim, partindo de determinada estimativa $\hat{\rho}_{g}$, pode-se inferir sobre um número de repetições a partir do qual não seria mais compensador aumentá-lo. Como os valores das estimativas de $\hat{\rho}_{\mathrm{g}}$ (Tabela 5) aumentam do DIC para o DBC e deste para o látice, observa-se, por um lado, que, quanto mais aprimorado for o delineamento, menor será o número de repetições necessário para se atingir determinado limite e, por outro, que se pode atingir esse limite em qualquer delineamento, desde que com número adequado de repetições. Então, em um mesmo número de tratamentes, um DIC pode ser tão eficiente quanto um DBC ou látice, dependendo do número de repetições usadas em cada um.

É importante mencionar também que $\rho_{\bar{g}}$ pode ser usado no cômputo do progresso genético com seleção para determinado caráter sob seleção. Segundo Resende (2002), a expressão real para se estimar o progresso com seleção, quando o material genético vai ser usado no mesmo local onde se encontra o experimento, deve ser corrigida multiplicando-a por $\rho_{\bar{g}}$. 


\subsection{Caráter peso de massa verde}

As anovas, segundo os delineamentos DIC, DBC e látice, encontram-se nas Tabelas 6,7 e 8 , respectivamente. As estimativas de $\mathrm{E}_{\mathrm{b} / \mathrm{i}} ; \mathrm{E}_{1 / \mathrm{b}} ; \mathrm{c}^{2} ; \rho_{\mathrm{r}} ; \rho_{\mathrm{g}} ;$ e $\rho_{\overline{\mathrm{g}}}$ são apresentadas na Tabela 9.

Uma vez que a estimativa de F para blocos foi significativa (Tabela 6), pode-se inferir que a capacidade de teste do DBC foi boa. Entretanto, o valor $(14,3 \%)$ da estimativa da eficiência relativa $\hat{E}_{b / i}$ não foi tão alto, e o da correlação intraclasse $\hat{c}^{2}$ ficou acima

Tabela 6 - Análise de variância do caráter peso de massa verde em erva-mate com três anos de idade, considerando-se o delineamento inteiramente ao acaso (DIC)

Table 6-ANOVA for wet green leaf weight trait in maté at three year old in a completely randomized design (CRD)

\begin{tabular}{lcccr}
\hline FV & GL & QM & F & P(F) \\
\hline Famílias/Procedências & 59 & 43734,5 & 1,7 & $0,00 \%$ \\
Erro & 512 & 25490,7 & 2,5 & $0,00 \%$ \\
Dentro & 2579 & 10310,9 & & \\
\hline
\end{tabular}

$\overline{\mathrm{k}}=5,48 ; \hat{\sigma}_{\mathrm{d}}^{2}=56503,7 ; \hat{\sigma}^{2}=15179,8 ; \hat{\sigma}_{\mathrm{f}}^{2}=2027,1 ; \hat{\mathrm{h}}_{\mathrm{i}}^{2}=11,0 \%$

Tabela 7 - Análise de variância do caráter peso de massa verde em erva-mate com três anos de idade, considerando-se o delineamento em blocos ao acaso (DBC)

Table 7 - ANOVA for wet green leaf weight trait in maté at three year old in a randomized block design (RBD)

\begin{tabular}{lcccc}
\hline FV & GL & QM & F & P(F) \\
\hline Repetições & 8 & 226822,6 & 10,2 & $0,00 \%$ \\
Famílias/Procedências & 59 & 43734,5 & 2,0 & $0,00 \%$ \\
Erro & 504 & 22295,0 & 2,2 & $0,00 \%$ \\
Dentro & 2579 & 10310,9 & & \\
\hline
\end{tabular}

$\overline{\mathrm{k}}=5,48 ; \hat{\sigma}_{\mathrm{d}}^{2}=56503,7 ; \hat{\sigma}_{\mathrm{e}}^{2}=11984,1 ; \hat{\sigma}_{\mathrm{f}}^{2}=2382,2 ; \hat{\mathrm{h}}_{\mathrm{i}}^{2}=13,4 \% ; \hat{\sigma}_{\mathrm{r}}^{2}=3195,7$

de $10 \%$ (Tabela 9). Infere-se, portanto, que a eficiência, tanto relativa quanto absoluta, não foi tão satisfatória, ou seja, no caráter em questão, havia ainda considerável heterogeneidade dentro das repetições.

Quando foi considerada a estimativa $\hat{E}_{l b b}$ (Tabela 9), observou-se que a eficiência relativa do látice em relação ao DBC foi bem mais elevada. Associando esta última com a estimativa $\hat{c}^{2}$, concluiu-se que o látice manifestou
Tabela 8 -ANOVA do caráter peso de massa verde em ervamate com três anos de idade, seguindo-se o delineamento em látice

Table 8 -ANOVA for wet green leaf weight trait in maté at three year old in a lattice design

\begin{tabular}{lcccr}
\hline FV & GL & QM & F & P(F) \\
\hline Repetições & 8 & 226822,6 & 18,5 & $0,00 \%$ \\
Famílias/ & 59 & 58344,3 & 4,8 & $0,00 \%$ \\
Procedências (aj.) & & & & \\
Bloco/Repetições (aj.) & 63 & 92480,7 & 7,5 & $0,00 \%$ \\
Erro intra bloco & 441 & 12268,5 & 1,2 & $0,01 \%$ \\
Dentro & 2579 & 10310,9 & & \\
\hline
\end{tabular}

$\overline{\mathrm{k}}=5,48 ; \hat{\sigma}_{\mathrm{d}}^{2}=56503,7 ; \hat{\sigma}_{\text {eib }}^{2}=1957,6 ; \hat{\sigma}_{\mathrm{f}}^{2}=5119,5 ; \hat{\mathrm{h}}_{\mathrm{i}}^{2}=32,2 \% ; \hat{\sigma}_{\mathrm{b}}^{2}=10026,5$

boa capacidade de teste e boa eficiência. A eficiência do látice em relação ao DIC foi de 2,08 (1,143 x 1,817), portanto inferior àquela $(2,39)$ do caráter altura de plantas.

Observou-se também, com era esperado, que, à medida que cresciam os valores das estimativas das eficiências, cresciam também os das $\mathrm{h}_{\mathrm{i}}^{2}$ (Tabelas 2, 3, 4, 6,7 e 8), bem como as das correlações $\hat{\rho}_{\mathrm{g}} \mathrm{e} \hat{\rho}_{\overline{\mathrm{g}}}$ (Tabelas 5 e 9), em ambos os caracteres.

Algumas considerações em relação aos prováveis fatores responsáveis pela heterogeneidade na área onde foi conduzido o experimento podem ser discutidas. Nas análises de fertilidade do solo com relação às 12 variáveis (Tabelas 10e 11), não foram detectadas diferenças significativas entre as repetições em praticamente nenhuma delas (Tabela 10). Ou seja, em relação às características químicas do solo, que supostamente têm efeito sobre o desenvolvimento de plantas, não houve heterogeneidade entre as repetições. Como a estimativa do efeito de repetições foi altamente significativa nas duas características, a hipótese mais provável até o momento é de que esta se deva a diferenças de umidade entre as repetições, já que estavam em diferentes níveis.

As variáveis químicas analisadas apresentaram, entretanto, diferenças significativas entre estratos dentro das repetições (Tabela 11), e essa tendência foi em uma única direção, já que a interação entre estratos e repetições não foi significativa em nenhuma variável (anovas não apresentadas). O que se conclui, então, a partir dos dados da Tabela 11, é que houve piora do solo quanto aos aspectos químico e nutricional 
(redução de $\mathrm{pH}$, incremento de alumínio, redução de $\mathrm{Ca}+\mathrm{Mg}$ etc.) em todas as repetições, do estrato 1 (início das repetições) para o estrato 3 (final das repetições).

Observando as estimativas dos coeficientes de regressão da Tabela 12, constatou-se uma tendência significativa de incremento do valor quanto ao caráter altura de planta ao longo das repetições (de 1 a 4). Nas repetições 5 e 6, não se verificou nenhuma tendência significativa. Já nas repetições 7 a 9 essa tendência foi significativa, porém negativa (estimativas de b negativas). Com o caráter produção de massa verde aconteceu um comportamento muito parecido (Tabela 12). Essas informações corroboram os altos valores das estimativas de $\mathrm{El} / \mathrm{b}$ encontradas em ambas as características estudadas.

Embora variações nas propriedades químicas do solo possam influenciar muito o seu desempenho em erva-mate, tanto em condições de laboratório quanto de campo (REISSMANN et al., 1991; REISSMANN e PREVEDELLO, 1992; REISSMANN et al., 1997), não se pode atribuir as variações observadas nesses caracteres (Tabela 12) às variações nas propriedades químicas do solo dentro das repetições (Tabela 11). Essa hipótese poderia ser cogitada se as estimativas dos coeficientes de regressão (Tabela 12) fossem todas significativas e com o mesmo sinal. A hipótese mais provável para explicar as variações dentro das repetições são, novamente, variações na umidade do solo, já que há uma ligeira variação de nível dentro destas.

Tabela 9 - Estimativas de Eb/i; El/b; c2; $\rho$ r; $\rho \mathrm{g}$; e $\rho_{\bar{g}}$ do caráter peso de massa verde em erva-mate com três anos de idade Table 9 -Estimations for Eb/i; El/b; c2; $\rho r ; \rho g$; and $\rho_{\bar{g}}$ parameters for wet green leaf weight trait in maté at three year old

\begin{tabular}{lcccccc}
\hline & & \multicolumn{3}{c}{ Parâmetros estimados } \\
\hline Delineamentos & $\hat{\mathrm{E} b} / \mathrm{i}$ & $\hat{\mathrm{E}} \mathrm{l} / \mathrm{b}$ & $\hat{\mathrm{c}}^{2}$ & $\hat{\rho}_{\mathrm{r}}$ & $\hat{\rho}_{\mathrm{g}}$ & $\hat{\rho}_{\overline{\mathrm{g}}}$ \\
DIC & - & - & - & - & 0,074 & 0,418 \\
DBC & 1,143 & - & 0,169 & 0,125 & 0,097 & 0,491 \\
Látice & - & 1,817 & 0,031 & 0,450 & 0,294 & 0,790 \\
\hline
\end{tabular}

Tabela 10 - Estimativas de médias por repetição (R1 a R9) de algumas variáveis indicadoras de fertilidade do solo, na área experimental do ensaio com erva-mate

Table 10 - Mean estimations by repetition ( $R 1$ to $R 9)$, for some chemicals related to soil fertility, on an area where a maté trial was carried out

\begin{tabular}{|c|c|c|c|c|c|c|c|c|c|c|c|c|c|c|c|c|c|c|c|c|c|}
\hline \multicolumn{22}{|c|}{ Variáveis analisadas } \\
\hline Rep. & PH & & $\mathrm{Al}^{+++}$ & & $\mathrm{H}+\mathrm{Al}$ & & $\mathrm{Ca}+\mathrm{Mg}$ & & $\mathrm{Ca}^{+2}$ & & $\mathrm{~K}^{+}$ & & $\mathrm{T}$ & & $\mathrm{P}$ & & $\mathrm{C}$ & M\% & & $\mathrm{V} \%$ & \\
\hline R1 & 4,40 & B & 2,00 & $\mathrm{a}$ & 9,80 & a & 6,30 & a & 3,95 & $\mathrm{a}$ & 0,12 & & 16,22 & a & 5,50 & a & $24,52 \mathrm{ab}$ & 25,77 & $\mathrm{Ab}$ & 38,93 & \\
\hline 22 & 4,47 & $\mathrm{Ab}$ & 2,07 & a & 9,60 & a & 6,33 & a & 3,48 & a & 0,09 & 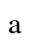 & 16,00 & a & 3,67 & $\mathrm{a}$ & $22,48 \quad b$ & 28,33 & $\mathrm{Ab}$ & 38,25 & \\
\hline 23 & 4,53 & $\mathrm{Ab}$ & 1,85 & a & 9,50 & a & 6,72 & a & 3,95 & a & 0,10 & $\mathrm{a}$ & 16,32 & a & 3,83 & a & $23,72 \mathrm{ab}$ & 25,35 & $\mathrm{Ab}$ & 40,07 & \\
\hline $\mathrm{R} 4$ & 4,60 & A & 1,45 & a & 8,92 & a & 7,72 & $\mathrm{a}$ & 4,82 & a & 0,10 & a & 16,73 & $\mathrm{a}$ & 5,83 & $\mathrm{a}$ & $27,37 \mathrm{ab}$ & 18,42 & $\mathrm{~B}$ & 45,18 & 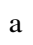 \\
\hline 25 & 4,50 & $\mathrm{Ab}$ & 1,90 & $\mathrm{a}$ & 9,53 & $\mathrm{a}$ & 6,72 & $\mathrm{a}$ & 4,35 & $\mathrm{a}$ & 0,09 & $\mathrm{a}$ & 16,33 & $\mathrm{a}$ & 3,83 & $\mathrm{a}$ & $26,93 \mathrm{ab}$ & 24,17 & $\mathrm{Ab}$ & 40,57 & a \\
\hline R6 & 4,48 & $\mathrm{Ab}$ & 2,12 & $\mathrm{a}$ & 9,78 & $\mathrm{a}$ & 4,52 & a & 2,75 & a & 0,10 & $\mathrm{a}$ & 14,40 & a & 3,17 & $\mathrm{a}$ & $27,17 \mathrm{ab}$ & 34,78 & A & 31,03 & $d$ \\
\hline 27 & 4,50 & $\mathrm{Ab}$ & 1,68 & $\mathrm{a}$ & 9,42 & $\mathrm{a}$ & 4,80 & a & 3,20 & $\mathrm{a}$ & 0,11 & $\mathrm{a}$ & 14,35 & $\mathrm{a}$ & 2,17 & $\mathrm{a}$ & $27,58 \mathrm{ab}$ & 33,65 & A & 31,55 & \\
\hline 8 & 4,55 & $a b$ & 1,78 & a & 9,67 & $\mathrm{a}$ & 5,12 & $\mathrm{a}$ & 3,35 & $\mathrm{a}$ & 0,09 & $a$ & 14,88 & $\mathrm{a}$ & 1,50 & $\mathrm{a}$ & 28,47 a & 31,68 & $\mathrm{Ab}$ & 32,78 & \\
\hline R9 & 4,63 & $\mathrm{a}$ & 1,25 & $\mathrm{a}$ & 9,08 & $\mathrm{a}$ & 5,05 & a & 3,23 & $\mathrm{a}$ & 0,08 & $a$ & 14,23 & $\mathrm{a}$ & 1,67 & $\mathrm{a}$ & $26,93 \mathrm{ab}$ & 22,58 & $\mathrm{Ab}$ & 34,93 & $\mathrm{a}$ \\
\hline DMS & 0,18 & & 0,96 & & 1,24 & & 3,74 & & 2,93 & & 0,03 & & 2,77 & & 4,96 & & 5,14 & 14,31 & & 13,83 & \\
\hline
\end{tabular}

Médias seguidas de letras diferentes têm mais de $95 \%$ de probabilidade de serem diferentes pelo teste de Tukey. 
Tabela 11 - Estimativas de médias por estrato (E1 a E3) de algumas variáveis indicadoras de fertilidade do solo, na área experimental do ensaio com erva-mate

Table 11 -Mean estimations by strip (St1 to St3), for some chemicals related to soil fertility, on an area where it was carried out a maté trial

\begin{tabular}{|c|c|c|c|c|c|c|c|c|c|c|c|c|c|c|c|}
\hline \multicolumn{16}{|c|}{ Variáveis analisadas } \\
\hline \multicolumn{2}{|c|}{ Estrato $\mathrm{PH}$} & $\mathrm{Al}^{+++}$ & & $\mathrm{H}+\mathrm{Al}$ & $\mathrm{Ca}+\mathrm{Mg}$ & $\mathrm{Ca}^{+2}$ & $\mathrm{~K}^{+}$ & $\mathrm{T}$ & $\mathrm{P}$ & & $\mathrm{C}$ & M\% & & $\mathrm{V} \%$ & \\
\hline E1 & $4,69 \mathrm{a}$ & 1,03 & $\mathrm{c}$ & $8,72 \mathrm{~b}$ & 8,33 a & 5,29 a & $0,10 \mathrm{a}$ & 17,15 a & 6,06 & $\mathrm{a}$ & $30,01 \mathrm{a}$ & 13,96 & $\mathrm{c}$ & 47,36 & $\mathrm{a}$ \\
\hline E2 & $4,56 \mathrm{~b}$ & 1,56 & $\mathrm{~b}$ & $9,18 \mathrm{~b}$ & $5,99 \mathrm{~b}$ & $4,06 \mathrm{a}$ & $0,10 \mathrm{a}$ & $15,27 \mathrm{~b}$ & 3,17 & $\mathrm{~b}$ & $26,23 \mathrm{~b}$ & 22,17 & $\mathrm{~b}$ & 39,18 & $\mathrm{~b}$ \\
\hline E3 & $4,31 \mathrm{c}$ & 2,78 & $\mathrm{a}$ & $10,53 \mathrm{a}$ & $3,44 \mathrm{c}$ & $1,67 \mathrm{a}$ & $0,09 \mathrm{~b}$ & $14,07 \mathrm{c}$ & 1,17 & $\mathrm{~b}$ & $22,15 \mathrm{c}$ & 45,45 & $\mathrm{a}$ & 24,56 & $\mathrm{c}$ \\
\hline DMS & 0,07 & 0,40 & & 0,53 & 1,58 & 1,24 & 0,01 & 1,17 & 2,10 & & 2,18 & 6,07 & & 5,86 & \\
\hline
\end{tabular}

Médias seguidas de letras diferentes têm mais de $95 \%$ de probabilidade de serem diferentes pelo teste Tukey.

Tabela 12 - Estimativas de coeficientes de regressão dos caracteres altura de planta e produção de massa verde em um ensaio com erva-mate

Table 12 - Regression coefficient estimations for plant height and wet green leaf weight at a maté trial

\begin{tabular}{|c|c|c|c|c|c|c|c|c|c|}
\hline \multicolumn{10}{|c|}{ Altura de planta } \\
\hline Repetições & 1 & 2 & 3 & 4 & 5 & 6 & 7 & 8 & 9 \\
\hline Coef. Regr.(b) & 0,326 & 0,139 & 0,159 & 0,158 & $-0,077$ & $-0,041$ & $-0,092$ & $-0,234$ & $-0,246$ \\
\hline Sgnificância & $0,00 \%$ & $1,77 \%$ & $0,38 \%$ & $0,10 \%$ & $10,66 \%$ & $47,10 \%$ & $2,98 \%$ & $0,00 \%$ & $0,00 \%$ \\
\hline \multicolumn{10}{|c|}{ Peso de massa verde } \\
\hline Repetições & 1 & 2 & 3 & 4 & 5 & 6 & 7 & 8 & 9 \\
\hline$\overline{\text { Coef. Regr.(b) }}$ & 12,418 & 4,940 & 3,290 & 2,069 & 1,607 & 3,228 & 0,001 & $-3,073$ & $-2,123$ \\
\hline Significância & $0,00 \%$ & $0,01 \%$ & $0,43 \%$ & $0,11 \%$ & $0,88 \%$ & $0,09 \%$ & $50,00 \%$ & $0,02 \%$ & $0,02 \%$ \\
\hline
\end{tabular}

Cada coeficiente de regressão de cada repetição foi estimado a partir de 64 pares de dados: a variável dependente foi a média do caráter na parcela e a independente, a posição (1 a 64) da referida parcela.

\section{CONCLUSÕES}

- A capacidade de teste do DBC foi satisfatória com relação ao caráter altura em plantas aos 12 meses de idade, mas insatisfatória quanto ao caráter produção de massa verde aos três anos, conclusão essa corroborada por outras estatísticas de interesse para o melhoramento, estimadas sob cada modelo.

- Quanto aos caracteres em estudo, a heterogeneidade do ambiente experimental mostrou-se útil na seleção de genótipos com maior plasticidade fenotípica, desde que monitorada por um modelo adequado.

- Em um mesmo número de tratamentos, quanto mais aprimorado for um delineamento menor será o número de repetições necessário para se atingir determinada eficiência, conforme previsões teóricas. Entretanto, com um número suficiente de repetições, um delineamento simples pode ser tão eficiente quanto outro mais aprimorado.

\section{REFERÊNCIAS BIBLIOGRÁFICAS}

COCHRAN, W.G.; COX, G.M. Experimental

designs. 2. ed. New York: John Wiley \& Sons, 1957.

CRUZ, C.D.; REGAZZI, A.J. Modelos

biométricos aplicados ao

melhoramento genético. Viçosa:

Universidade Federal de Viçosa, 1994.

FEDERER, W.T. Esperimental design. New York: Macmillan, 1955.

FERNANDES, J.S.C. et al. Estimativas de parâmetros relacionados ao melhoramento genético da erva-mate: possibilidade de seleção precoce. Scientia Agrária, v.1, p.45-53, 2000. 
FU, Y. et al. Incomplete block design for genetic testing: statistical efficiencies of estimating family means. Canadian Journal of Forest Research, v. 28, p.977-986, 1998.

PIMENTEL GOMES, F. Curso de estatística experimental. 14. ed. São Paulo: Nobel, 2000.

RAMALHO, M.A.P.; FERREIRA, D.F.; OLIVEIRA, A.C. Experimentação em genética e melhoramento de plantas. Lavras: Universidade Federal de Lavras, 2000.

REISSMANN, C.B.; PREVEDELLO, B.M.S. Influência da calagem no crescimento e na composição química da erva-mate (Ilex paraguariensis St. Hil.). Revista do Instituto de Floresta de São Paulo, v.4, p.265-269, 1992.

REISSMANN, C.B. et al. Production and foliar N, $\mathrm{P}, \mathrm{K}, \mathrm{Ca}$ and $\mathrm{Mg}$ lvels in erva-mate (Ilex paraguariensis St. Hil.) related to increasing base saturation levels. Arquivos de Biologia e Tecnologia, v.40, p.241-249, 1997.

REISSMANN, C.B. et al. Suscetibilidade da ervamate à clorose induzida pela calagem. Revista do Setor de Ciências Agrárias, v.11, p.273-278, 1991.
RESENDE, M.D.V. Genética biométrica e estatística no melhoramento de plantas perenes. Brasília: Embrapa Informação Tecnológica, 2002. 975p.

RESENDE, M.D.V.; FERNANDES, J.S.C. Análises alternativas envolvendo procedimento BLUP associado ao delineamento experimental de blocos incompletos ou látice. Revista de

Matemática e Estatística, v.18, p.103-124, 2000.

VENCOVSKY, R.; BARRIGA, P. Genética biométrica no fitomelhoramento. Ribeirão Preto: SBG, 1992.

VIEIRA, S.; HOFFMANN, R. Estatística experimental. São Paulo: Atlas, 1989.

WILLIAMS, E.; MATHESON, A.C.

Experimental designs and analisys for use tree improvement. Victoria: CSRIO, 1994.

WINGE, H. et al. Apresentação. In: ERVAMATE: Biologia e cultura no cone sul, 1., 1992, Porto Alegre. Porto Alegre: Universidade Federal do Rio Grande do Sul, 1995. p.11-13. 